\title{
Expression and diagnostic value of proteins in Mycobacterium tuberculosis
}

\author{
Z.Y. Zhu' ${ }^{1}$, Y. Liu ${ }^{2}$, H.B. Wang', J.Z. Xiao' ${ }^{1}$ Y.F. Qiu ${ }^{3}$, L. Yan ${ }^{3}$, D. Chen ${ }^{3}$, \\ A.G. Liu $^{3}$ and X. Yang ${ }^{3}$ \\ ${ }^{1}$ Hainan Provincial Nongken General Hospital, Haikou, Hainan, China \\ ${ }^{2}$ College of Environment and Plant Protection of Hainan University, Haikou, \\ Hainan, China \\ ${ }^{3}$ Nanjing Potomac Biotechnology Co. Ltd., Nanjing, Jiangsu, China \\ Corresponding author: Z.Y. Zhu \\ E-mail: zhuzhongyuan197@163.com
}

Genet. Mol. Res. 13 (3): 7780-7790 (2014)

Received July 24, 2013

Accepted March 18, 2014

Published September 26, 2014

DOI http://dx.doi.org/10.4238/2014.September.26.16

\begin{abstract}
We constructed a prokaryotic expression vector expressing the Mycobacterium tuberculosis protein TB16.3, as well as 3 other proteins, including TB15.3, CFP-10, and Rv2626C, which were purified and analyzed for their effectiveness as detection antibodies. The TB16.3 genes of M. tuberculosis H37Rv genomic DNA were amplified by polymerase chain reaction, inserted into the expression vector pET-30a, and expressed in Escherichia coli. An enzyme-linked immunosorbent assay was used to detect the $4 M$. tuberculosis antibodies. Engineered E. coli bacteria expressing TB16.3 and the 3 other proteins were constructed and found mainly to be soluble. For recombinant TB16.3 proteins, serum samples of 118 tuberculosis (TB) patients and 96 healthy controls were analyzed. Sensitivity, specificity, and adjusted concordance rate for the TB16.3 antibody were $72.9,86.5$, and $79.6 \%$, respectively. The positive rate of Rv2626C antibody in TB patients $(44.1 \%)$ was significantly lower than that in normal controls $(75.0 \%$, $\left.\chi^{2}=20.8, \mathrm{P}<0.01\right)$. TB15.3 and TB16.3 were used for simultaneous detection and showed sensitivity, specificity, and repeatability rates of
\end{abstract}


$69.4,96.9$, and $83.7 \%$. The antibody positive rate and specificity for patients with lung disease was 9.6 and $90.4 \%$, respectively. TB15.3 and TB16.3 were mixed and detected simultaneously. Combined with the results for TB15.3, the sensitivity, specificity, and concordance rates were $82.2,95.9$, and $88.9 \%$, respectively. The concordance rate was the highest value observed. Target genes were cloned into a host strain and expressed successfully. The TB16.3 recombinant protein may be used as a new serological antigen for tuberculosis diagnosis.

Key words: Mycobacterium tuberculosis; TB16.3 protein; Prokaryotic expression vector; Serological diagnosis

\section{INTRODUCTION}

Tuberculosis (TB), which is caused by Mycobacterium tuberculosis, is one of the most infectious diseases and currently has one of the highest mortality rates of any disease. It has been estimated that TB infection affects one-third of the global population, with 8.9-9.9 million new TB cases reported annually. Approximately 1.3 million people die of the disease. Thus, TB is a global public health problem (WHO, 2010). The most critical aspect of TB prevention and control is early detection and correct medication. Methods for early diagnosis of TB are urgently needed. Sputum smear detection of acid-fast bacilli remains the preferred method of TB diagnosis and therapy assessment in many developing countries. However, the limitations of this method include its low sensitivity, multiple testing, and loss of manpower, among others (Steingart et al., 2006). Recently, serological methods for TB diagnosis have been applied, and early laboratory diagnosis of TB has achieved good results; however, several limitations remain (Zhu et al., 2004; Steingart et al., 2009; Ben-Selma et al., 2011). Identification of stable and efficient specific antigens or antigen combinations has been challenging. Thus, the development of satisfactory antigens and antigen combinations with high sensitivity and specificity has become a focus in the serological diagnosis of TB. In this study, TB16.3 was examined for its ability to detect TB and Bacille Calmette-Guérin (BCG) genetic variation. Using genetic engineering to directly express the antigen, the serological diagnostic effectiveness of TB16.3 was analyzed. TB16.3 is not purified from M. tuberculosis culture filtrate. We cloned and expressed TB16.3 as well as 3 other proteins (Liu et al., 2011; Zhang et al., 2011) to detect TB antibodies in human serum.

\section{MATERIAL AND METHODS}

\section{Strains and vectors}

M. tuberculosis H37Rv, Escherichia coli BL21 (DE3) cells, and the pET-30 expression vector were stored in the clinical laboratory department of our hospital.

\section{Reagents}

Isopropyl $\beta$-D-1-thiogalactoside (IPTG) and kanamycin (Kan) were purchased from Shanghai Sangon Biological Engineering Technology \& Services Ltd. (Shanghai, China). 
DNA polymerase, T4 DNA ligase, restriction endonucleases, and the DNA marker were purchased from Takara Co., Ltd. (Shiga, Japan). The pre-stained protein marker was purchased from Beijing Bai Tai Ke Biotechnology Co., Ltd. (Beijing, China). An agarose gel DNA extraction kit was purchased from Beijing Tian Gen Biotechnology (Beijing, China). Columns for 6x His-tagged Ni-NTA Agarose protein purification were obtained from QIAGEN (Hilden, Germany). Common high-purity biochemical reagents were purchased from Biosharp (Hefei, China), and other reagents were of analytical grade. Horseradish peroxidase-labeled anti-human immunoglobulin (Ig) was purchased from Solarbio (Beijing, China). The chromogenic substrate was purchased from InTec Technology, Ltd. (Xiamen, China).

\section{Serum source}

Serum from 118 diagnosed TB patients was collected from the Haikou Tuberculosis Control Department, including serum from 83 males and 35 females. Twenty patients had smear-positive samples and 98 patients had smear-negative samples. Sixty-six patients were $\leq 40$ years old, 29 patients were aged $41-59$ years, and 23 patients were $\geq 60$ years old. Fiftythree patients did not have TB but had other lung diseases; 12 patients had community-acquired pneumonia, 26 patients had chronic obstructive pulmonary disorder, 8 patients had lung infection, and 6 patients had lung cancer. All were hospital inpatients. Serum from 96 normal controls was collected from healthy subjects.

\section{Primers}

According to the gene sequence of TB16.3 in standard strains of M. tuberculosis H37Rv (GenBank sequence No. CAD97060; Garnier et al., 2003), the following primers were synthesized: for the 5' end (TB16.3F), 5'-CGGAATTCATGGCGGACAAGACGACACA-3', and for the 3' end (TB16.3R), 5'-CCGCTCGAGTCAGCCCTCGACTCGTTTCT-3'. Underlined bases indicate EcoRI and $X h o \mathrm{I}$ restriction sites. Primers were synthesized by GenScript, Ltd. (Piscataway Township, NJ, USA).

\section{TB16.3 gene amplification and cloning}

H37Rv genomic DNA was used as a template, and TB16.3 gene fragments were amplified by polymerase chain reaction (PCR). The reaction conditions were as follows: $95^{\circ} \mathrm{C}$ for $5 \mathrm{~min}, 95^{\circ} \mathrm{C}$ for $30 \mathrm{~s}, 53^{\circ} \mathrm{C}$ for $30 \mathrm{~s}$, and $72^{\circ} \mathrm{C}$ for $1 \mathrm{~min}$, for a total of $35 \mathrm{cycles}$. After this, the temperature was held at $72^{\circ} \mathrm{C}$ for 7 min and then maintained at $4^{\circ} \mathrm{C}$. PCR products were purified using an Agarose DNA extraction kit (Tian Gen Biotechnology, Beijing, China). After restriction enzyme digestion, the fragments were ligated into the expression plasmid pET30a using T4 DNA ligase. Ligation products were transformed into E. coli BL21 (DE3) competent cells, and positive recombinants were selected for sequencing by Nanjing GenScript Biological Engineering Co., Ltd. (Nanjing, China).

\section{Induced expression of target proteins}

Sequence-verified recombinants were inoculated in Luria-Bertani (LB) liquid me- 
dium containing $50 \mathrm{mg} / \mathrm{L} \mathrm{Kan}$. After shaking the culture at $37^{\circ} \mathrm{C}$, the absorbance at $600 \mathrm{~nm}$ $\left(\mathrm{A}_{600}\right)$ was adjusted to $0.6-0.8$. IPTG was added to reach a final concentration of $1 \mathrm{mM}$, the culture was incubated at $37^{\circ} \mathrm{C}$ for $1 \mathrm{~h}$ from the start of induced expression, and the samples were collected at 1-h intervals until $6 \mathrm{~h}$ of expression. The bacterial cells were harvested for sodium dodecyl sulfate-polyacrylamide gel electrophoresis (SDS-PAGE) analysis.

\section{Expression and purification of recombinant proteins}

The bacterial cells were collected following inducible expression, sonicated, and centrifuged to remove cell debris. The pellet and supernatant were collected. The SDS-PAGE analysis was used for target protein expression. The QIAGEN 6 x His-tagged Ni-NTA Agarose protein purification column was used for the purification of His-tagged recombinant proteins.

\section{Detection of recombinant TB16.3 protein antigens by enzyme-linked immunosorbent assay (ELISA)}

Using the matrix method, the optimum antigen coating concentration and the secondary antibody dilution times were determined. The optical density at a wavelength of $450 \mathrm{~nm}$ $\left(\mathrm{OD}_{450}\right)$ was measured using a microplate reader. $\mathrm{OD}_{450}$ values $\geq 2.1$ times the negative control value were defined as positive based on previously described methods (Liu et al., 2011; Zhang et al., 2011).

\section{Statistical analysis}

Sensitivity, specificity, positive and negative predictive values, and diagnostic accuracy were calculated based on methods described in the literature (Lv et al., 1991). Statistical significance was evaluated using the chi-square test, with $\mathrm{P}<0.05$ considered to be statistically significant. The SPSS18.0 statistical package was used for analysis (SPSS, Inc.; Chicago, IL, USA).

\section{RESULTS}

\section{Construction and identification of the recombinant plasmid}

The TB16.3 gene was successfully amplified from H37Rv genomic DNA. The length was 435 base pairs (bp), which was consistent with the design. The constructed expression vectors pMD-19T-TB16.3 and pET-30a-TB16.3 were digested using restricted enzymes after purification. The 435-bp fragments are displayed in Figure 1A and B. Sequencing results showed that the sequence of the gene inserted into the vector was $100 \%$ consistent with the reported sequence (Garnier et al., 2003).

\section{Optimization of expression conditions}

A 37-kDa protein band was observed on the SDS-PAGE gel after IPTG induction (Figure 2A). Maximum expression was observed $5 \mathrm{~h}$ after induction (Figure 2B). Inducible expression occurred at $28^{\circ}, 37^{\circ}$, and $42^{\circ} \mathrm{C}$. Although the TB16.3 protein was expressed at the 
3 different temperatures, the expression reached a maximum at $37^{\circ} \mathrm{C}$ (Figure $3 \mathrm{~A}$ ). Analysis of expression at 3 different concentrations of IPTG showed that $1 \mathrm{mM}$ IPTG most effectively induced protein expression (Figure 3B).

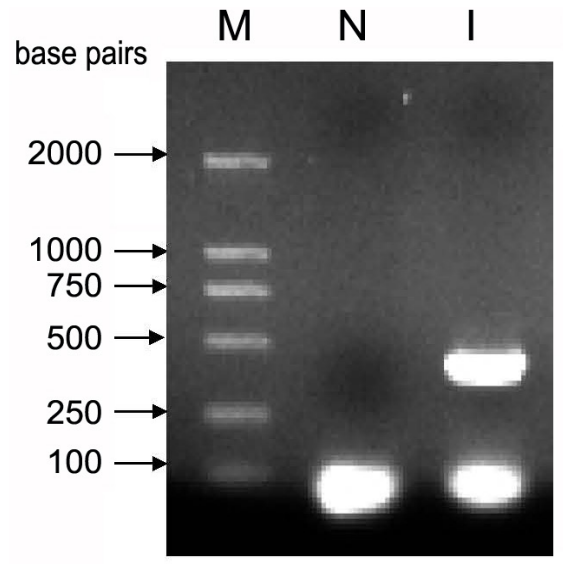

A

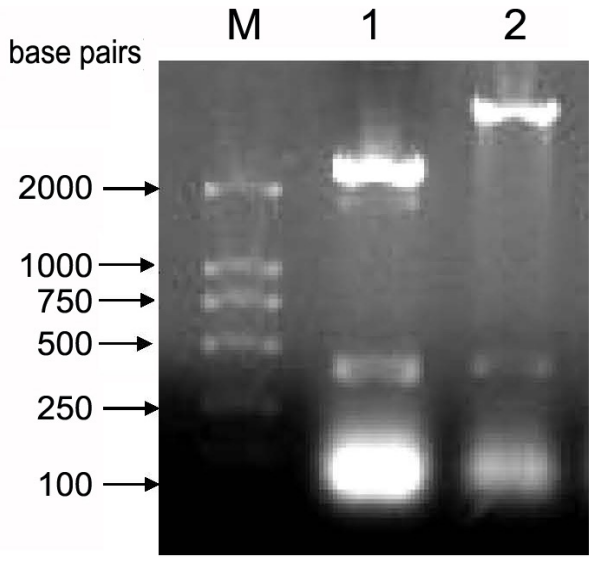

B

Figure 1. PCR amplification of the target band and restructuring pMD-19T with pET-30a plasmid digestion results. A. Lane $M=$ DNA marker; lane $N=$ negative control; lane $I=\mathrm{TB} 16.3 \mathrm{PCR}$ product $(435 \mathrm{bp})$; $\mathbf{B}$. Lane $M=\mathrm{DNA}$ marker; lanes 1 and 2 = pMD-19T-TB16.3 and pET-30a-TB16.3 after double-digestion of the double bar belt.

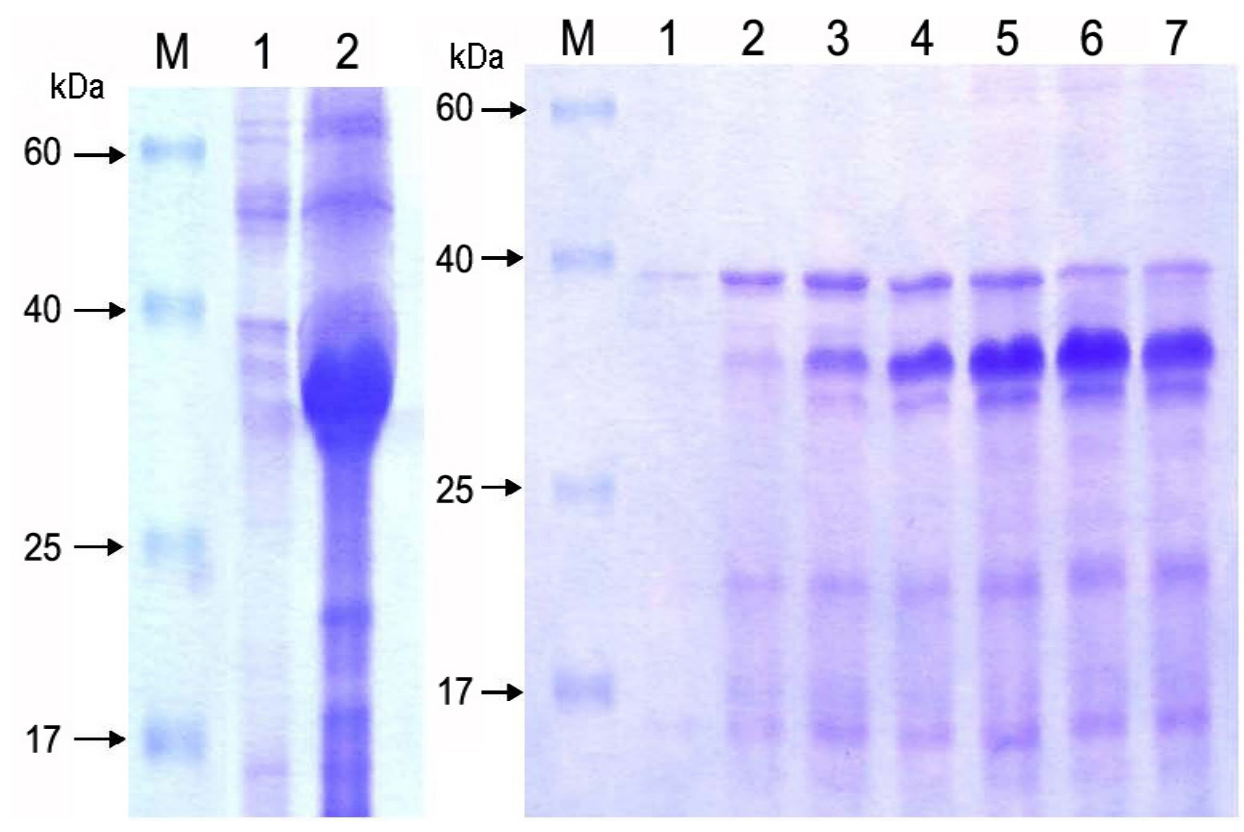

Figure 2. Protein expression of antigen and induction time gradient results. Lane $M=$ pre-stained protein marker; A. lane 1 = uninduced engineered bacteria; lane $2=$ engineered bacteria induced after $4 \mathrm{~h}$; B. lane $1=$ uninduced engineered bacteria; lanes 2-7 = 1-6 $\mathrm{h}$ induced engineered bacterial culture supernatant. 


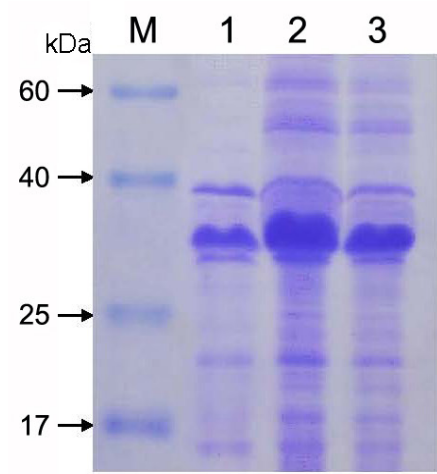

A

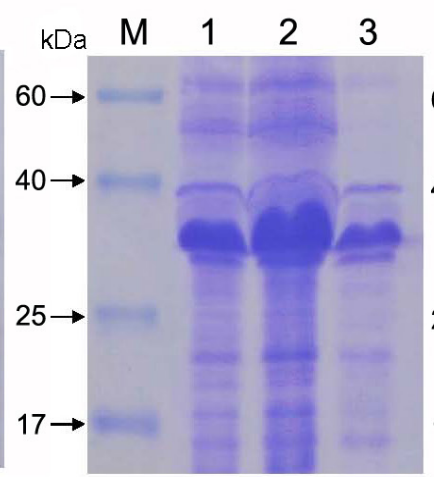

B

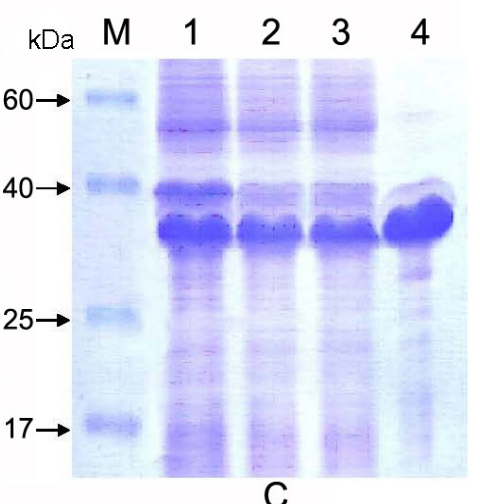

Figure 3. Induced expression optimization results. Lane $M=$ pre-stained molecular weight marker; A. lanes $1-3=$ cloned bacteria at $28^{\circ}, 37^{\circ}$, and $42^{\circ} \mathrm{C}$ induction results. B. lanes $1-3=0.5$ and $1.5 \mathrm{mM}$ IPTG for TB16.3 antigen expression. C. Lane 1 = engineered bacterium culture supernatant; lane 2 = engineered bacterium broken precipitation; lane $3=$ engineered bacterium broken supernatant; lane $4=$ purified rTB16.3 peptides.

\section{Soluble analysis and purification results of TB16.3 peptides}

While the highest expression was observed at $37^{\circ} \mathrm{C}$ (Figure 3B), the recombinant antigens were mainly present in inclusion bodies. When the temperature was reduced to $28^{\circ} \mathrm{C}$, the recombinant antigens were mainly in the form of soluble protein. Therefore, we used a temperature of $28^{\circ} \mathrm{C}$ to expand fermentation. The supernatants were collected after expression, and the TB16.3 peptides were purified using a nickel agarose gel purification column to obtain highly purified TB16.3 (Figure 3C).

\section{Establishment of anti-rTB16.3 detection technology using ELISA}

Using matrix methods, the optimum coating concentration of recombinant TB16.3 peptides (rTB16.3) was found to be $0.1 \mu \mathrm{g} / \mathrm{well}$. The optimum working concentration of mixed antigens was $0.1 \mu \mathrm{g} / \mathrm{well}$. The content of the 2 recombinant proteins was $0.05 \mu \mathrm{g} /$ well. The optimum working concentration of horseradish peroxidase secondary antibody was 1:1000.

\section{Detection of TB using TB16.3}

The results of TB16.3 protein-coating and serum results for TB patients using ELISA are shown in Table 1 and Figure 4. TB patients with sputum smear-positive results showed the highest TB16.3 sensitivity. The positive rate of Rv2626C antibody in TB patients $(44.1 \%)$ was significantly lower than that in normal controls $\left(75.0 \%, \chi^{2}=20.8, \mathrm{P}<\right.$ $0.01)$. CFP-10 and TB16.3 showed higher sensitivity. Without the sputum smear test, the CFP-10 antibody positive rate was the highest. The sensitivities of detection using TB15.3, TB16.3, and TB15.3 + TB16.3 are shown in Figure 4; their specificities were 99.0, 96.9, and $86.5 \%$, respectively. 


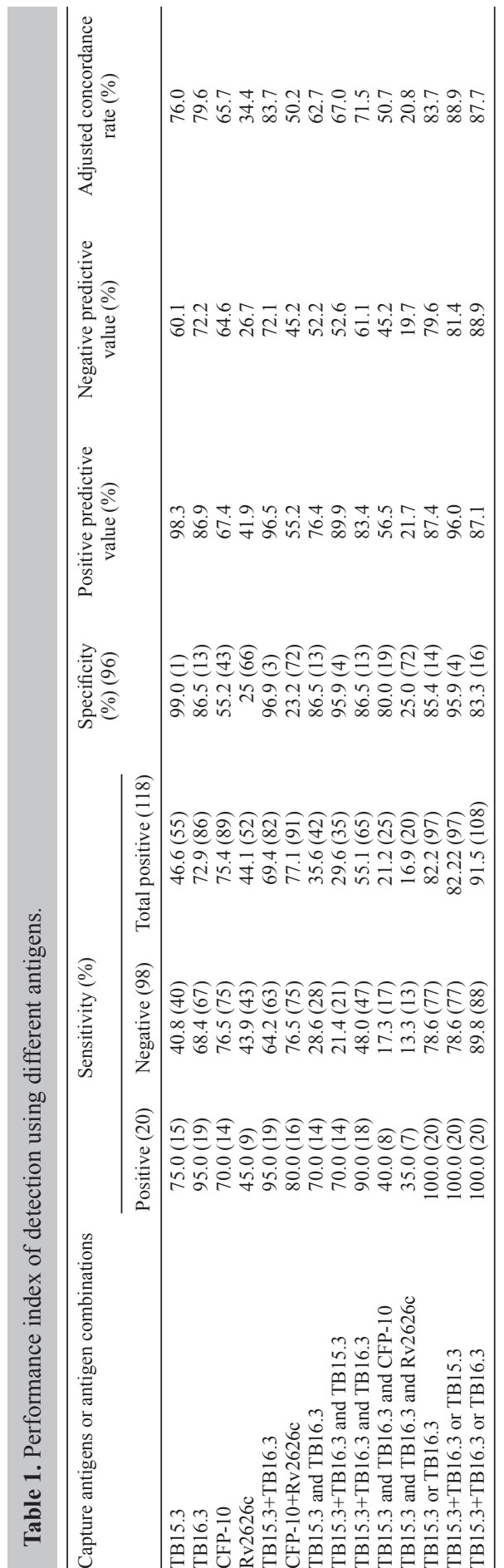



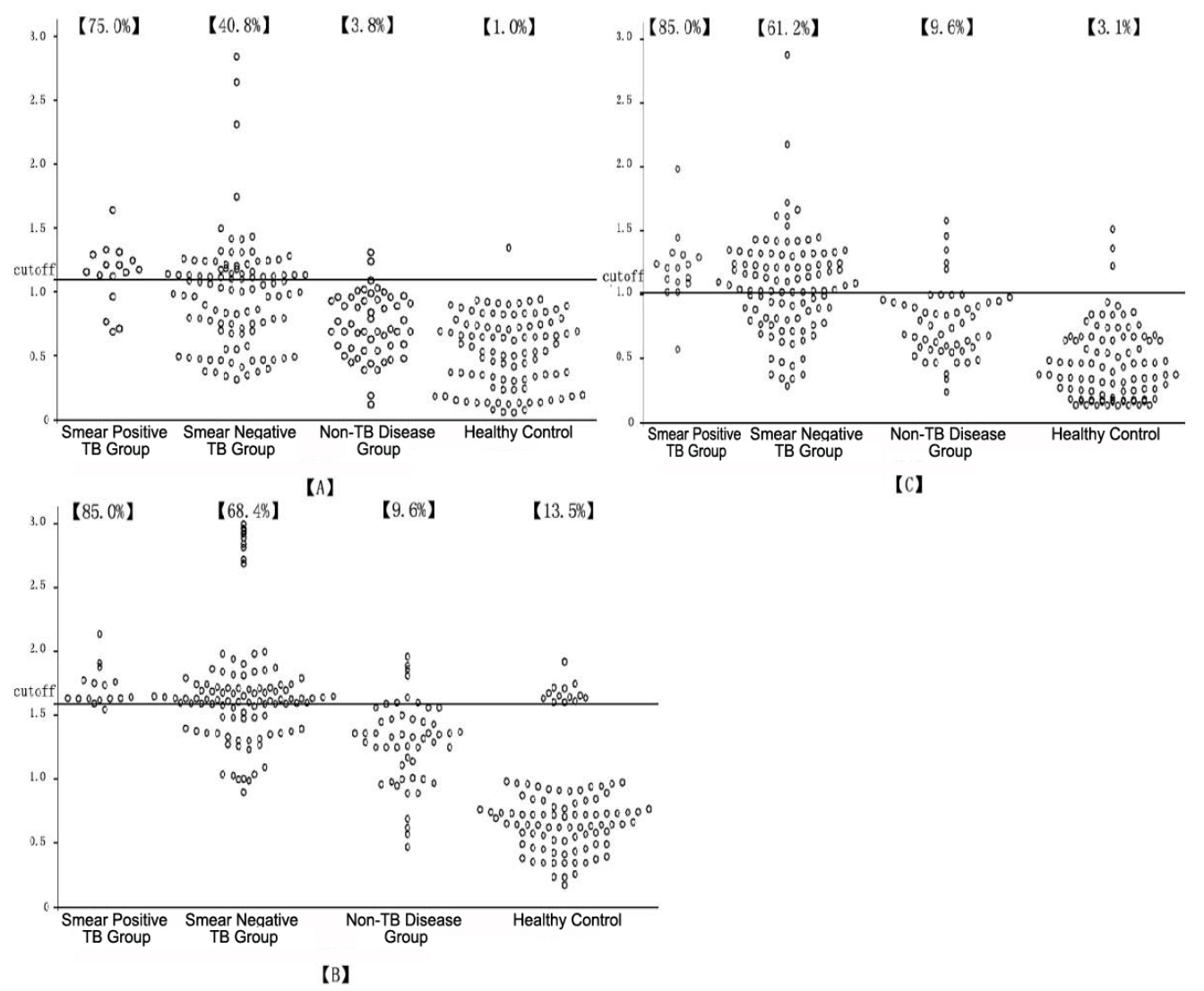

Figure 4. Tuberculosis patients' TB15.3 and TB16.3 antibody results by ELISA. A. B. and C. show antibody sensitivity and specificity using ELISA in smear-positive, smear-negative TB patients, the non-TB acute disease group, and healthy serum for anti-TB15.3, anti-TB16.3, and anti-TB15.3 + TB16.3.

\section{Four recombinant antigen combinations in $\mathrm{TB}$ antibody detection}

The results of the combined antigen ELISA test of serum in TB patients are shown in Table 1. An equal mixture of Rv2626C and CFP-10 was detected, with seropositive rates very similar between TB patients and normal controls (77.1 and 76.8\%, respectively). If TB15.3, TB16.3, and Rv2626C were detected, the positive results of the 3 was defined as positive; the positive rates of TB patients and controls were $16.9 \%(20 / 118)$ and $75.0 \%(72 / 96)$, respectively $\left(\chi^{2}=72.8, \mathrm{P}<0.001\right)$. This difference was very significant, indicating that the Rv2626C antibody can be used as an indicator of improvement in patients.

The data in Table 1 also show that TB15.3 and TB16.3 were detected at the same time, with sensitivity, specificity, and consistency rates of $69.4,96.9$, and $76.0 \%$, respectively. TB15.3 and TB16.3 were both detected individually as well. If either of the 2 antibody results were positive, the test results were judged as positive. The sensitivity, specificity, and consistency rates were $82.2,85.4$, and $83.7 \%$, respectively. If a mixture of TB15.3 and TB16.3 
was detected, the test results or TB16.3 individual test results were defined as positive; the sensitivity, specificity, and consistency rates were $91.5,83.3$, and $87.7 \%$, respectively. If the mixture of TB15.3 and TB16.3 was detected, the test results or TB15.3 individual test results were defined as positive; the sensitivity, specificity, and consistency rates were 82.2, 95.9, and $88.9 \%$, respectively. The concordance rate was the highest value among antigen or antigen cocktail tested.

\section{DISCUSSION}

Laboratory diagnosis of TB is crucial to controlling the disease. Early detection of row bacteria in patients and early treatment can prevent the spread of TB. Significantly blocking the spread of multi-drug-resistant TB is even more important. The means of diagnosing TB in the laboratory currently include the following. First, direct or enriched smear microscopy can intuitively discover pathogens, with the drawback of a low positive rate (Steingart et al., 2006). Second, TB culture in vitro can improve positive rates; however, although susceptibility testing and species identification can be performed, this test is time-consuming, taking as long as 6-8 weeks. Liquid culture techniques can significantly shorten the time to diagnosis, but TB patients typically cannot afford the expensive equipment and reagents. Third, molecular biology techniques are used for TB diagnosis, which can improve sensitivity and show high specificity, and even detect resistance genes. However, the high false-positive results and the high expense of such tests may prevent the widespread use of this method (Perkins and Cunningham, 2007). Fourth, immunological diagnostic methods are used, which can be divided into cellular immunity and humoral immunity diagnostic methods. Cell immunological diagnostic methods, such as ELISPOT and TB-IGRA tests, are only suitable for TB screening in countries with low TB incidence. Because the false positive diagnosis rate is high and the tests are costly, prospects for applying this method in developing countries appear to be limited.

In TB-specific antibody detection, the immunological tests are fast and simple, with no specific equipment or facilities required. These tests can be performed for a large population. Tuberculosis antibody detection technology primarily includes ELISA, colloidal gold filtration assay, and protein chip technology (Zhu et al., 2004). The use of antigens has shifted from purified proteins of mycobacterial culture to genetically engineered antigens. The currently used TB antigens include the 38-kDa protein (Silva et al., 2003; Ben-Selma et al., 2011), the 16-kDa protein (Senol et al., 2007; Ben-Selma et al., 2011), lipoarabinomannan, HspX, MTB48 (Lodes et al., 2001), and Mtb81 (Silva et al., 2003; Perkins et al., 2003; Ben-Selma et al., 2011). Despite significant research efforts, the sensitivity, specificity, sputum acid-fast bacilli-positive concordance rate, and relevance of a serious condition reveal little useful information. Positive antibodies are not useful as a primary method for diagnosing TB. Therefore, screening for TB proteins is used in antibody detection, which is currently an important area of study.

The TB16.3 protein is encoded by the TB gene $R v 2815 C$, producing a mature protein of 144 amino acids with a molecular weight of $16.3 \mathrm{kDa}$; thus, the protein was named TB16.3. Because it cannot be separated from the H37Rv protein filtrate or cell detritus, it has been referred to as a hypothetical protein (Garnier et al., 2003). The amino acid sequence is identical to that in Mycobacterium bovis and is similar to the ML0889 protein expressed in Mycobacterium leprae (Garnier et al., 2003). Sable et al. (2005) used ion-exchange chromatography 
and high-resolution gel separation technology to purify 104 peptide chains. In the detection of peptide chains with a molecular weight of $16.9 \mathrm{kDa}, \mathrm{Rv} 2185 \mathrm{C}$ was considered to be the expression product that stimulates the patients' peripheral blood lymphocytes to produce higher levels of interferon (IFN)- $\gamma$. The ELISA method can be used to detect TB, suggesting that TB16.3 can stimulate the body to produce TB-specific cellular and humoral immunity. Gaseitsiwe et al. (2010) used the peptide chip (peptide microarray) to analyze TB16.3 epitopes. They found that of the 44 12-amino acid peptide segments in the protein, 5, 2, and 1 could bind with DR1, DR2, and DR4 in MHCII antigens, respectively. This accounted for 18\% (8/44) of the epitopes. The positive rate was among the highest in the $61 \mathrm{~TB}$ proteins tested. Weldingh et al. (2005) cloned and expressed TB16.3, which was used as a capture protein. An antibody test based on an ELISA showed that the sensitivity was $44-55 \%$ in Danish TB patients. The sensitivity was $88-98 \%$ in TB patients in Africa, while the specificity reached $97 \%$. This indicated that TB16.3 could be used as a diagnostic protein.

In this study, the TB16.3 gene $R v 2185 C$ was PCR-amplified from H37Rv genomic DNA, and an expression vector was constructed. Sequencing and restriction digestion indicated the proper construction of the expression vector. The molecular weight of TB16.3 was found to be $37 \mathrm{kDa}$ using SDS-PAGE, which differs from the theoretically predicted size. Some of this increase in size might be due to the inserted amino acids and His-tag used in constructing the expressed vector, but the increase might also be associated with the protein band structure or charge abnormalities. The data in Table 1 show the reactivity of TB16.3 and TB serums, and indicate that we expressed the correct protein. Through induction under different conditions, we confirmed that the expression vector could express the TB16.3 protein; we also determined optimum preparation and purification conditions for the protein.

The results showed that using an established ELISA method for detecting TB in serum, TB16.3, which we cloned and expressed, showed an antibody positive rate of $72.9 \%$ and specificity of $86.5 \%$. The sensitivity rate was higher than that of the Danish anti-TB16.3 antibody-positive rate as reported by Weldingh et al. (2005). However, the sensitivity and specificity rate was lower than the antibody-positive rate in the African population. This may be due to the strength of the immune response is inconsistent in different regions or ethnicities. We also observed that the Rv2626C antibody-positive rate in normal subjects was significantly higher than that in patients with TB. The TB-positive rate was $16.9 \%$, which was lower than that in normal subjects in which the positive rate was $75.0 \%$. The Rv2626C protein may not be expressed in TB-infected non-TB patients or TB-recovered patients. Further studies are necessary to determine whether the protein with the corresponding antibodies may be used as a recovery indicator in these patients.

Our data reflect that the expressed proteins, particularly TB16.3 and TB15.3, can be used to improve detection sensitivity after mixing coating detection for joint or separate detection. This test can be used to determine whether TB patients are discharging bacteria. Thus, using existing tests, a combination of TB protein antibody detection was found to be valuable for diagnosing TB. This method can also be used for our listed products upgrading (Zhu et al., 2004), making the product more competitive.

\section{ACKNOWLEDGMENTS}

Research supported by grants from Hainan key technology projects in 2008 (\#080209). 


\section{REFERENCES}

Ben-Selma W, Harizi H and Boukadida J (2011). Immunochromatographic IgG/IgM test for rapid diagnosis of active tuberculosis. Clin. Vaccine Immunol. 18: 2090-2094.

Garnier T, Eiglmeier K, Camus JC, Medina N, et al. (2003). The complete genome sequence of Mycobacterium bovis. Proc. Natl. Acad. Sci. U. S. A. 100: 7877-7882.

Gaseitsiwe S, Valentini D, Mahdavifar S, Reilly M, et al. (2010). Peptide microarray-based identification of Mycobacterium tuberculosis epitope binding to HLA-DRB1*0101, DRB1*1501, and DRB1*0401. Clin. Vaccine Immunol. 17: 168-175.

Liu Y, Zhu ZY and Wang HB (2011). Mycobacterium tuberculosis TB15.3 protein expression and purification. Zhong Guo Re Dai Yi Xue 11: 1301-1302.

Lodes MJ, Dillon DC, Mohamath R, Day CH, et al. (2001). Serological expression cloning and immunological evaluation of MTB48, a novel Mycobacterium tuberculosis antigen. J. Clin. Microbiol. 39: 2485-2493.

Lv HQ, Liu XH, Qian ZS and Hu CW (1991). Basic methods for clinical researchers. Hu Bei Ke Xue Ji Shu Chu Ban She, Wu Han 270-275.

Perkins MD and Cunningham J (2007). Facing the crisis: improving the diagnosis of tuberculosis in the HIV era. J. Infect. Dis. 196 (Suppl 1): S15-S27.

Perkins MD, Conde MB, Martins M and Kritski AL (2003). Serologic diagnosis of tuberculosis using a simple commercial multiantigen assay. Chest 123: 107-112.

Sable SB, Kumar R, Kalra M, Verma I, et al. (2005). Peripheral blood and pleural fluid mononuclear cell responses to low-molecular-mass secretory polypeptides of Mycobacterium tuberculosis in human models of immunity to tuberculosis. Infect. Immun. 73: 3547-3558.

Senol G, Erer OF, Yalcin YA, Coskun M, et al. (2007). Humoral immune response against 38-kDa and 16-kDa mycobacterial antigens in tuberculosis. Eur. Respir. J. 29: 143-148.

Silva VM, Kanaujia G, Gennaro ML and Menzies D (2003). Factors associated with humoral response to ESAT-6, 38 kDa and $14 \mathrm{kDa}$ in patients with a spectrum of tuberculosis. Int. J. Tuberc. Lung Dis. 7: 478-484.

Steingart KR, Ng V, Henry M, Hopewell PC, et al. (2006). Sputum processing methods to improve the sensitivity of smear microscopy for tuberculosis: a systematic review. Lancet Infect. Dis. 6: 664-674.

Steingart KR, Dendukuri N, Henry M, Schiller I, et al. (2009). Performance of purified antigens for serodiagnosis of pulmonary tuberculosis: a meta-analysis. Clin. Vaccine Immunol. 16: 260-276.

Weldingh K, Rosenkrands I, Okkels LM, Doherty TM, et al. (2005). Assessing the serodiagnostic potential of 35 Mycobacterium tuberculosis proteins and identification of four novel serological antigens. J. Clin. Microbiol. 43: 57-65.

World Health Organization (WHO) (2010). Global Tuberculosis Control. World Health Organization, Geneva.

Zhang D, Zhu ZY and Wang HB (2011). Mycobacterium tuberculosis protein CFP-10 Cloning and purification. Zhong Guo Re Dai Yi Xue 11: 1180-1181.

Zhu ZY, Wang HB and Liu AG (2004). Mycobacterium tuberculosis multiple antigen protein microarray detection. Zhong Guo Re Dai Yi Xue 4: 907-910. 